\title{
The Outcome of Vaginal Birth After Caesarean Section (VBAC): A Descriptive Study
}

\author{
Rahman $\mathrm{R}^{1}$, Khanam $\mathrm{NN}^{2}$, Islam N ${ }^{3}$, Begum $\mathrm{KF}^{4}$, Pervin $\mathrm{HH}^{5}$, Arifuzzaman $\mathrm{M}^{6}$
}

\begin{abstract}
The study, conducted in the tertiary care hospital of Dhaka Bangladesh, describes the outcome of vaginal birth after caesarean section (VBAC) in women with a previous caesarean.
\end{abstract}

A prospective study was carried out from 1st January 2007 to 31st December, 2007 on 126 women with one prior lower segment cesarean section (LSCS) for a nonrecurrent cause. All unbooked women and those with estimated fetal weight more than $3.5 \mathrm{~kg}$, breech presentation, history of postoperative wound infection after previous LSCS, anemia $(\mathrm{Hb}<10 \mathrm{gm} \%)$, pregnancy induced hypertension, diabetes, heart disease, renal disease, cephalopevic disproportion abnormal presentation and placenta praevia were excluded from the study. An informed consent was taken for allowing a trial of vaginal delivery. Spontaneous onset of labor was awaited up to 41 weeks. Induction of labor was considered only in highly selected cases. Labor was constantly supervised by competent staff and meticulously monitored by cardiotocography (CTG).

Out of the 126 women enrolled for the study, 26 had to leave the station leaving a total of 100 patients; 72 patients underwent elective repeat C/S, 28 patients (28\%) of these underwent trial of labour, among them 15 had successful vaginal delivery (53.57) but 13 patients failed the attempt and had to undergo emergency caesarean section. To assist in the 2nd stage of labour, 6 had ventouse application. In total 85 cases needed repeat caesarean section. Among the cases there was one case of scar dehiscence (6.6\%), one case of cervical tear (6.6\%), two cases of manual removal of placenta (13.3\%), one case of post partum hemorrhage (6.6) and one case of puerperal pyrexia (6.6).Perinatal

1. Corresponding Author:

Dr. Reefaat Rahman MBBS, FCPS, MCPS

Medical Officer, Department of Obstetrics and Gynaecology Bangabandhu Sheikh Mujib Medical University, Dhaka

2. Dr. Nurun Nahar Khanam MBBS, DGO, FCPS, MS Associate Professor, Department of Obstetrics and Gynaecology Bangabandhu Sheikh Mujib Medical University, Dhaka

3. Dr. Nilufar Islam MBBS, MS Medical Officer, Department of Obstetrics and Gynaecology Bangabandhu Sheikh Mujib Medical University, Dhaka

4. Dr. Kazi Farhana Begum MBBS, FCPS Medical Officer, Department of Obstetrics and Gynaecology Bangabandhu Sheikh Mujib Medical University, Dhaka

5. Dr. Hasna Hena Pervin MBBS, FCPS Medical Officer, Department of Obstetrics and Gynaecology Bangabandhu Sheikh Mujib Medical University, Dhaka

6. Dr. Md. Arifuzzaman MBBS, MS Junior Consultant Ispahani Islamia Eye Institute \& Hospital, Dhaka morbidity was comparable with the elective repeat $C / S$ group.

VBAC should be considered in cases of previous one cesarean delivery for nonrecurrent indication.

Key words: vaginal birth after cesarean delivery, previous cesarean delivery.

\section{Introduction}

Caesarean section has been a part of human culture since ancient times and there are tales in both western and eastern cultures of this procedure resulting in live mothers and off springs. Numerous references to caesarean section appear in ancient Hindu Egyptians, Greecians, Romans and other European folklore ${ }^{1}$. In past 20 years, the rate of $\mathrm{C} / \mathrm{S}$ has steadily increased from about $5 \%$ to more than $20 \%{ }^{25}$. The policy- once a caesarean always a caesarean is no longer tenable. A planned vaginal birth after a previous $\mathrm{C} / \mathrm{S}$ should be recommended for women whose first c/s was by lower segment transverse incision and who have no other indication for $\mathrm{C} / \mathrm{S}$ in present pregnancy ${ }^{22,23,26}$.

There is a definite risk of uterine rupture in vaginal birth after cesarean delivery (VBAC) often leading to catastrophes which can be avoided by rapid diagnosis and prompt intervention. Evidence confirming the safety of VBAC within proper guidelines has been available for more than 10 years ${ }^{11,12,13,15}$. However, wide variations in VBAC rates still exist between hospitals and physicians. The present study was undertaken to reascertain these facts with the hope that more women will be encouraged to avoid an unnecessary repeat cesarean section by opting for vaginal delivery. VBAC offers distinct advantages over a repeat cesarean section since the operative morbidity and mortality are completely eliminated, the hospital stay is much shorter and expenses involved are much less ${ }^{14,16,17,24}$. The rate of cesarean section needs to be reduced and this can be achieved to a small extent by avoiding primary cesarean sections done without explicit indications and more importantly by resorting to a trial of vaginal delivery after previous cesarean section which is safe for the fetus ${ }^{2-5}$. The purpose of this study was to evaluate the efficacy and safety of VBAC.

\section{Materials \& Methods}

A prospective study was carried out on 100 women with one previous lower segment cesarean section (LSCS) for a nonrecurrent cause, from 1st January, 2007 to 31st December 2007. All the cases were booked in the antenatal clinic and were regularly reporting for check up. The following cases were excluded from the study - 
1. Associated medical disorder like anemia $(\mathrm{Hb}<10 \mathrm{gm}$ $\%$ ), pregnancy induced hypertension, diabetes, heart disease and renal disease

2. Estimated fetal weight $>3.5 \mathrm{~kg}$

3. Breech presentation

4. History of postoperative wound infection following previous LSCS

5. Details of the previous cesarean operation not available

6. Contraindications to vaginal delivery like cephalopelvic disproportion, major degree placenta praevia, and transverse lie.

7. Postdated pregnancy with unfavorable cervix

All women were admitted if they went into spontaneous labor. Those who failed to go into labor on their own were induced after completion of 41 weeks. Induction was started in the morning with 5 units of oxytocin in $500 \mathrm{ml}$ of $5 \%$ glucose and increased gradually from $6 \mathrm{mIU} / \mathrm{minute}$ to a maximum of $36 \mathrm{mIU} / \mathrm{minute}$ with the aim of getting 3-4 uterine contractions every 10 minutes each lasting 40-45 seconds. Whether the labor was spontaneous or induced it was monitored with

1. Hourly recording of vital parameters - temperature, pulse, respiration and blood pressure

2. Continuous electronic fetal monitoring by cardiotocogrpahy

3. Monitoring of uterine contractions

4. Partograph

5. A close watch for the early recognition of scar dehiscence by identifying maternal tachycardia in absence of fever, vaginal bleeding, scar tenderness, and fetal heart rate alterations.

Attempt at vaginal delivery was abandoned if there was any suspicion of scar dehiscence or sign of fetal distress or unsatisfactory progress of labor. Vacuum extraction was used to cut short the second stage.

\section{Results}

Out of the total of 126 women recruited for the study, 26 dropped out. Of the remaining 100 women 5 went into preterm labor, 20 went into spontaneous labor between 37 and 40 weeks. Three women had to be induced since they did not go into spontaneous labor till 41 weeks. Demographic profile of the women is given in Table 1. It has been observed that women belonging to 20-30 age group had maximum successful vaginal delivery as shown in Table 2, indication for previous caesarean section, fetal distress was the commonest cause.

Table 1. Demographic profile $(n=100)$.

\begin{tabular}{lcccc}
\hline Maternal age & No of cases & Successful VD & $\begin{array}{r}\text { Emergency } \\
\text { repeat C/S }\end{array}$ & $\begin{array}{c}\text { Elective } \\
\text { Repeat C/S }\end{array}$ \\
\hline$<20$ & 8 & 2 & 2 & 7 \\
$20-30$ & 67 & 10 & 9 & 50 \\
$30-35$ & 16 & 2 & 1 & 10 \\
$35-40$ & 9 & 1 & 1 & 5 \\
Total & 100 & 15 & 13 & 72 \\
\hline
\end{tabular}

Table 2: indication for previous caesarean section, fetal distress was the commonest cause.

Indication for previous caesarean delivery

\begin{tabular}{lcc}
\hline & No & Percentage \\
\hline Fetal distress & 64 & $64 \%$ \\
Dystocia & 20 & $20 \%$ \\
Breech & 4 & $4 \%$ \\
Transverse lie & 01 & $1 \%$ \\
Placenta praevia & 03 & $3 \%$ \\
Abruptio placenta & 1 & $1 \%$ \\
Elderly primi & 2 & $2 \%$ \\
Severe pregnancy induced & 4 & $4 \%$ \\
hypertension & & \\
Cord Prolapse & 01 & $1 \%$ \\
\hline
\end{tabular}

Table 3 shows the mode of delivery among the 28 patients who underwent trial of labour.13 amongst 28 needed emergency repeat c/s, 9 patients had spontaneous, unassisted vaginal delivery, 6 patients needed vacuum extraction to cut short the second stage of labour.

Table 3. Mode of delivery in patients who underwent trial of labour $(n=28)$.

\begin{tabular}{lcc}
\hline Mode of delivery & Number & Percentage \\
\hline Spontaneous and unassisted & 9 & $32.14 \%$ \\
Vacuum extraction & 6 & 21.42 \\
Forceps delivery & 0 & 0 \\
Emergency repeat C/S & 13 & $46.428 \%$ \\
\hline
\end{tabular}

Table 4 shows the indications of emergency repeat caesarean section after failed trial. It shows that scar tenderness was the commonest cause followed by fetal distress.

Table 4. Indications for emergency repeat cesarean section after failed trial $(n=13)$

\begin{tabular}{lcc}
\hline Parameter & Number & Percentage \\
\hline Fetal distress & 4 & $30.76 \%$ \\
Scar tenderness & 6 & $46.15 \%$ \\
Failed progress of labour & 2 & $15.38 \%$ \\
Cervical dystocia & 01 & $7.6 \% \%$ \\
\hline
\end{tabular}

Table 5 shows the comparison of maternal complications in vaginally delivered group and repeat caesarean group. It can be seen that postnatal complications like puerperal pyrexia, blood transfusion, operative bladder injury and pulmonary edema were more common in repeat caesarean group.

One case of cervical tear occurred with ventouse extraction. Scar dehiscence was noticed in a case taken up for emergency LSCS due to scar tenderness. One case of primary atonic postpartum hemorrhage was managed with intravenous fluids, uterine massage, methergin injection, and misoprostol. 
Table 5. Maternal complications in vaginally delivered group and in repeat caesarean group

In vaginally delivered $\operatorname{group}(n=15)$ -

\begin{tabular}{lll}
\hline Parameter & Number & Percentage \\
\hline
\end{tabular}

Scar dehiscence after delivery

followed by hysterectomy

Puerperal pyrexia

$01 \quad 6.66 \%$

Cervical tear

$01 \quad 6.66 \%$

Manual removal of placenta

1

$6.66 \%$

02

$13 \%$

Primary atonic post partum

haemorrage

01

$6.6 \%$

In repeat caesarean group $(n=85)$ -

Wound infection requiring

secondary suture

Puerperal pyrexia

Blood transfusion required

Operative bladder injury

Spinal headache

Pulmonary edema

$\begin{array}{cc}7 & 8.23 \% \\ 4 & 4.7 \% \\ 8 & 9.41 \% \\ 1 & 1.17 \% \\ 1 & 1.17 \% \\ 1 & 1.17 \%\end{array}$

Table 6 compares the neonatal complications in vaginal deliveries and repeat caesarean group. Some neonatal complications like birth asphyxia, neonatal infection were more in repeat caesarean section then in vaginally delivered group

Table 6. Neonatal complications in vaginal deliveries $(n=15)$ and repeat caesarean group $(n=85)$

In vaginally delivered group

\begin{tabular}{lcc}
\hline Parameters & Number & Percentage \\
\hline Stillbirth & 1 & $6.6 \%$ \\
Birth asphyxia & 01 & $6.66 \%$ \\
Neonatal septicaemia & 02 & $13.33 \%$ \\
Neonatal jaundice & 02 & $13.33 \%$
\end{tabular}

Noenatal complications in repeat caesarean group

\begin{tabular}{lll} 
Stillbirth & 1 & $1.17 \%$ \\
Neonatal death & 1 & $1.17 \%$ \\
Birth asphyxia & 5 & $5.88 \%$ \\
Neonatal jaundice & 5 & $5.88 \%$ \\
Neonatal infection & 4 & $4.7 \%$ \\
\hline
\end{tabular}

\section{Discussion}

It is generally accepted that vaginal delivery is associated with lower maternal morbidity and mortality as against caesarean section. The morbidity associated with successful vaginal birth is about one-fifth that of elective caesarean. Perinatal risk is more after a failed trial of labour compared to elective repeated caesarean section without labour ${ }^{18,19}$. Failed trials of labour, with subsequent caesarean section involve almost twice the morbidity of elective section. The information is important for counseling women about their choices of delivery after a previous caesarean section. The adverse events include chorioamnionitis, postpartum endometritis, and uterine rupture requiring hysterectomy, blood transfusion, perinatal and neonatal deaths and neonatal neurological impairment. Many of these adverse events seen in trial of scar are attributable to the failure of labour and the requirement for a repeated emergency caesarean section. However, in this study there were fewer complications noted in those who underwent VBAC then elective or emergency repeat $\mathrm{C} / \mathrm{S}$. This study represents our observations for a period of 1 year. The selection of women for VBAC is mainly influenced by woman's desire and conditions favorable for vaginal delivery. The objective of this study was to evaluate the success rate and safety of attempted VBAC, in a tertiary care setting, after one previous cesarean delivery. In general, our institution offers a conservative approach both in the selection of women and in the management of their labor. Generally speaking women belonging to higher socioeconomic status were either not keen for VBAC or opted out of the study. Further, women with unfavourable cervix who had gone beyond their due date and had to be induced with PGE2 gel combined with oxytocin were abandoned from the study. In the present study, suitable women were selected for VBAC during early pregnancy after a thorough assessment, and adhering to strict inclusion and exclusion criteria as mentioned earlier. Of the 100 women, $15(15 \%)$ delivered vaginally and $85(85 \%)$ had to be taken up for emergency LSCS for various indications as given in (Table 4) All the six women who had one previous vaginal delivery, delivered vaginally in the present study. This is in line with the fact that the history of a previous normal vaginal delivery is the single most important predictor for a successful VBAC ${ }^{6,7}$ Farmer $^{8}$ and Turner ${ }^{9}$ have highlighted that caution is to be exercised in inducing labor in these patients because of the relatively higher risk of scar dehiscence and rupture associated with induction ${ }^{10,20,21}$. Induction was withheld till 41 weeks in our study for this reason. No case of scar dehiscence occurred in any of the 3 cases who underwent induction under close supervision. The maternal complications and perinatal morbidity in the present study are identical to those seen with other normal vaginal deliveries with the exception of scar dehiscence in one case $(6.66 \%)$.

The study shows the high success of VBAC and the fewer complications. Many women in the study were multiparous with a prior vaginal birth. Prior vaginal birth is a good predictor for the outcome of VBAC. An attempt for VBAC is well justified for post caesarean pregnancies with nonrecurrent indications. Screening for this should preferably begin at antenatal booking itself to minimize the associated risks. Proper selection, appropriate timing and suitable methods of induction with close supervision by competent staff are the key factors to achieve greater degree of success. 


\section{References}

1. Khotaba S, Volfson M, Tarazova L. Induction of labour in women with previous cesarean section using the double balloon device. Acta Obstet Gynecol Scand 2001;80:1041-2.

2. Porreco RP. Meeting the challenge of the rising cesarean birth rate. Obstet Gynecol 1990;75:133-6.

3. Pridjian G, Hibbard JU, Moawad AH. Cesarean : Changing the trends. Obstet Gynecol 1991;77:195-200.

4. Sachs BP, Kobelin C, Castro MA. The risks of lowering the cesarean delivery rate. N Engl J Med 1999;340:54-7.

5. Wing DA, Paul RH. Vaginal birth after cesarean section: selection and management. Clin Obstet Gynecol 1999;42:836-48.

6. Caughey AB, Shipp TD, Repke JT et al. Trial of labor after cesarean delivery: the effect of previous vaginal delivery. Am J Obstet Gynecol 1998;179:938-41.

7. Flamm BL, Geiger AM. Vaginal birth after cesarean delivery: an admission scoring system. Obstet Gynecol 1997;90:907-10.

8. Farmer RM, Kirschbaum T, Potter D. Uterine rupture during trial of labor after previous cesarean section. Am J Obstet Gynecol 1991;165:996-1001.

9. Turner MJ. Delivery after one previous cesarean section. Am J Obstet Gynecol 1997;176:741- 4.

10. Pathadey, Vanwoerdone. Induction of labor after a previous caesarean section, a retrospective study $\mathrm{J}$ Obstet Gynecol 2005;25:662.

11. Zeteroglu S, Ustun Y, Engin-Ustun Y, Sahin HG, Kamaci M. Eight years' experience of uterine rupture cases. J Obstet Gynaecol 2005;25:458-461.

12. Kayani SI, Alfirevic Z. Uterine rupture after induction of labor in women with PCS. BJOG 2005;112:451.

13. McDonagh MS, Osterweil P, Guise JM. The benefits and risks of inducing labour in patients with prior caesarean delivery: a systematic review BJOG 2005;112:1007-15.
14. Dunn C, O'Herlihy. Comparison of maternal satisfaction following vaginal delivery after caesarean section and caesarean section after previous vaginal delivery. European Journal of Obstetrics \& Gynecology and Reproductive Biology 2005;121:56-60.

15. Hassan A. Trial of scar and VBAC. J Ayub med coll Abbottabad 2005; 17:57.

16. Sur S, Mackenzie IZ. Does discussion of possible scar rupture influence preferred mode of delivery after CS B JOG 2005;25:338.

17. Macones GA, Peipert J, Nelson DB. Maternal complications with vaginal birth after cesarean delivery: a multicenter study. Am J Obstetrics Gynecology 2005;193:1656-62.

18. Quinones JN, Stamilio DM, Pare E. The effect of prematurity on VBAC success and maternal morbidity ACOG 2005; 105:519.

19. Huang WH, Nakashima DK, Rumney PJ, Keegan KA. Inter-delivery interval and the success of VBAC. ACOG 2002;99:41-44.

20. Juhasz G, Gyamfi C, Gyamfi P, Tocce K, Stone JL. Effect of body mass index and excessive weight gain on the success of VBAC. OG 2005;106:741.

21. Goodall, Ahn J, Chapa J, Hibbard J. Obesity as a risk factor for failed trial of labor in patients with previous cesarean delivery. Am J Obstetrics Gynecology 2005;192:1423-26.

22. SOGC clinical practice guidelines for VBAC Catalin S, Buhimschilrnia A, Patel BS, Andrew M, P.Weiner MCP. Rupture of the uterine scar during term labour: contractility or biochemistry? BJOG 2005;112:38.

24. Paré E, Quiñones JN, Macones GA. Vaginal birth after caesarean section versus elective repeat caesarean section: assessment of maternal downstream health outcomes. Int J Gyn Obst 2005;89:319.

25. Mukherjee SN. Rising caesarean section rate -Review article. J obstet gynaecol India 2006;56:298-300.

26. WORLD HEALTH ORGANISATION. Appropriate technology for birth. Lancet 1985;436-7. 